\title{
(3) A New Concept of Human Adult Glomerulonephritis
}

\author{
Seiichi SHrbata, M. D. \\ The Third Department of Internal Medicine, \\ Faculty of Medicine, University of Tokyo
}

\section{The accepted theory on the pathogenesis of diffuse glomerulonephritis}

Glomerulonephritis is now in general considered as a representative example of immune complex disease.

Glomerular diseases in man are provisionally classified as examples of immune complex disease or anti-glomerular basement membrane (GBM) antibody disease.

The finding of characteristic staining pattern for immunoglobulins and complement indicates that many forms of glomerulonephritis are immune complex disease and a few are due to anti-GBM antibodies.

In addition, it is considered that many, perhaps most, forms of glomerulonephritis have an immunologic basis.

The presumption that the disease is due to immune complexes is in general done in conditions in which the deposits are quite characteristic (i. e. conspicuous irregular and discontinuous deposits of immunoglobulins and complement components) and closely resemble those seen in experimental models.

\section{Critical aspects for the accepted theory}

Proof that a disease is due to deposition of immune complexes has been said to be satisfactorily accomplished only in cases of lupus nephritis, possibly poststreptococcal and malaria glomerulonephritis.

However, we can not find the similarities with morphologic changes of human lupus nephritis in a laboratory model which was presented by Tan et al. ${ }^{1)}$ : rabbit immunized with ultraviolet irradiated DNA (UV-DNA) produced high titers of serum antibody, but experimental renal disease induced by UV-DNA-anti UV-DNA immune complexes was only glomerulitis sometimes associated with focal proliferative lesions. Immunofluorescence showed rabbit $\operatorname{IgG}$ and $\beta_{1} \mathrm{C}$ in glomeruli only located in both mesangial areas and peripheral capillary loops.

Thus, proof that a disease is due to deposit of immune complex is not yet established even in lupus nephritis.

In addition, it is well established that immunofluorescent "linear" or "mesangial" pattern occurred prior to the appearance of membranous glomerulonephritis, and granular and lumpy deposits of immunoglobulin and complement were associated 
with massive proteinuria and histologic evidence of membranous glomerulonephritis ${ }^{2}$.

Thus, the granular deposits in cases of lupus nephritis can be said to result from the deposition of circulating antigen (DNA)-antibody (anti-DNA) complexes along the glomerular basement membrane, however this immune complex mechanism may be not a basic pathogenesis of lupus nephritis, but only an accelerating factor for the development of conspicuous membranous glomerulonephritis.

Samely, typical linear staining for IgG does not always represent anti-GBM antibody.

Koffler et al. have described linear staining in cases of lupus nephritis, but were unable to demonstrate anti-GBM antibody in eluates from glomeruli ${ }^{3}$.

Recently, Gallo have reported four patients with bright linear staining for IgG from whose kidneys anti-GBM antibody could not be eluted and also from whose peripheral blood serum anti-GBM antibody could not be demonstrated. Two of the patients had diabetic glomerulosclerosis ${ }^{4}$.

There is one glomerular disease which at present can be recognized through immunofluorescence observations. This is a condition which is referred as nephropathy with mesangial $\mathrm{IgG}$ deposits.

We can find these conditions in biopsy materials of many of human adult chronic glomerulonephritis.

Thus, summarizing the present status of investigations, various immunofluorescence staining patterns, which were found in the well known laboratory models, are utilized (only mechanically) as a morphologic indicator for interpretation of the development-mechanism of human diseases.

However, considering this method from the point of view of clinical nephrology, many unusual cases have been pointed out.

These results seem to require the appearance of the third experimental model other than Masugi nephritis and serum sickness (acute and chronic).

\section{Demonstration of a new laboratory model of human adult glomerulonephritis}

We have recently presented a new laboratory model of human adult glomerulonephritis. This model is referred to as glycoprotein or glycopeptide-nephritis.

a) Experiments using crude kidney homogenate

As shown by white arrow in Fig. 1, it is well known that Masugi produced nephrotoxic nephritis using crude kidney homogenate. On the other hand, as shown by dotted line of the same figure (Fig. 1), a single injection of crude kidney homogenate (low speed supernate of the homologous crude kidney homogenate) failed to produced renal disease.

Many attempts have been made to extract a water-soluble nephrotoxic antigen from kidney homogenate but these have always been unsuccessful.

b) Experiments using renal glycoprotein

The antigenic substance was highly purified by us. It was a water-soluble 


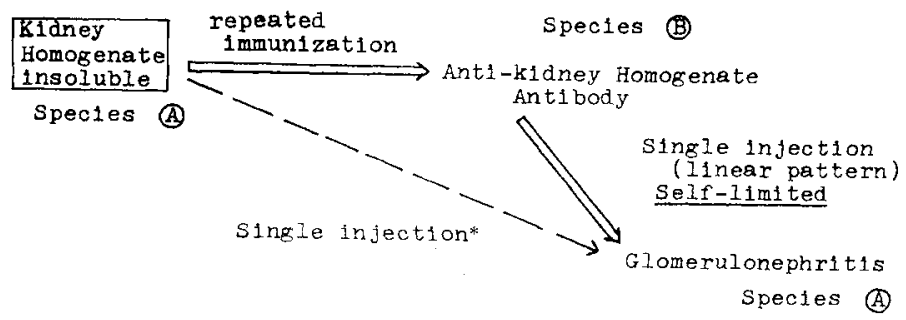

* Repeated immunization: Nephrotic Syndrome (Heyman's nephrosis)

(not proliferative glomerulonephritis)

Fig. 1. Masugi nephritis.

glycoprotein from rat GBM by the use of zone-electrophoresis and of gel-filtration using Sephadex G $200^{5,6,7)}$.

White arrow of Fig. 2 shows that a fatal glomerulonephritis, which is remarkably similar to the various stages of glomerulonephritis (including chronic glomerulonephritis) in man, was induced in rats by a single injection of antiserum against this substance ${ }^{8}$.

Furthermore, as shown by black arrow in this Fig. 2, rats that were given a single injection in the hind footpads of homologous water-soluble renal glycoprotein and Freund's incomplete adjuvant developed typical proliferative glomerulonephritis ${ }^{9}$.

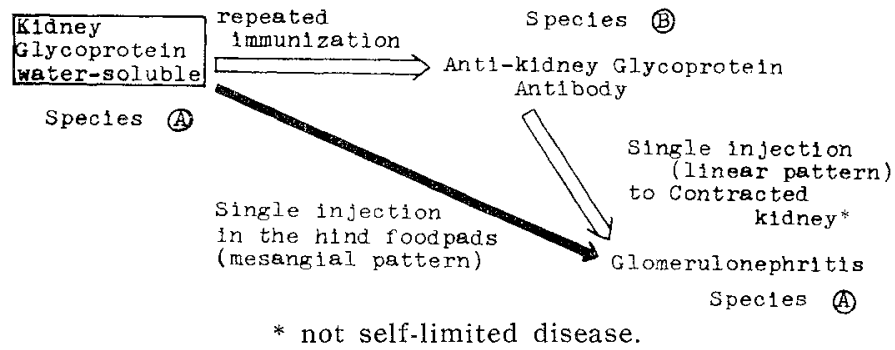

Fig. 2. Masugi nephritis and glycoprotein nephritis.

Proteinuria and morphologic changes of glomerulonephritis continued until death over twelve months. Immunofluorescent staining pattern of the glomerular capillary walls was so-called "mesangial pattern".

Inoculation of rats with as little as $5 \mu \mathrm{g}$ of the glycoprotein in Freund's complete adjuvant effectively induced typical proliferative glomerulonephritis. The nephritis could be transfered to previously healthy recipients with the eluate obtained from nephritic kidneys ${ }^{93}$.

c) Experiments using renal glycopeptide

As the next step of purification, renal glycopeptide was isolated from the above described nephritogenic glycoprotein by further exhaustive proteolytic digestion with pronase $^{10\rangle}$. 


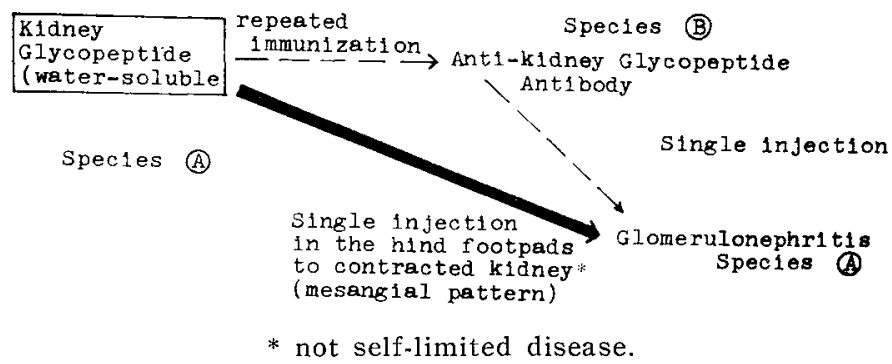

Fig. 3. Masugi nephritis and glycopeptide nephritis.

As shown by dotted line in Fig. 3, antisera against this nephritogenic glycopeptide no longer contained kidney-fixing antibodies, that is, we failed to produce nephrotoxic nephritis using this glycopeptide.

On the contrary, clinical and morphologic changes strikingly similar to human membranous glomerulonephritis were induced in all rats killed 4 to 5 months after injection, by a single inoculation in the hind footpads of homologous renal glycopeptide and Freund's incomplete adjuvant (black arrow of Fig. 3).

Regular and granular deposition of $\gamma$-globulin and $\beta_{1} \mathrm{C}$ took place along the GBM, besides the mesangial deposits of protein.

Analysis of the course of this experimental membranous glomerulonephritis revealed that proliferation and swelling of mesangial cells occurred prior to the appearance of typical membranous glomerulonephritis.

d) Experiments using the slightly modified glycopeptides

It is well known that by the use of ion-exchange chromatography (DEAE-cellulose) or TCA-treatment free amino acids or peptides can easily be separated from the active glycopeptide.

By the use of the material slighly modified (by ion-exchange chromatograph or TCA-treatment) from the nephritogenic glycopeptide which has an ability to induce membranous glomerulonephritis by one shot, we presented data on the successful production of typical morphologic changes of extracapillary and chronic glomerulonephritis (contracted kidney) strikingly similar to those of human adult glomerulonephritis (Fig. 4, lower) ${ }^{11,12}$.

It is of particular interest that active glycopeptide separated from free peptides by TCA-treatment or ion-exchange chromatograph (DEAE-cellulose), can induce typical morphologic changes of the whole spectrum (from the onset to the end-stage of diffuse glomerulonephritis), resembling that of human adult glomerulonephritis. The immunofluorescent staining of the kidney was of "mesangial pattern" throughout.

It seems clear that the difference between both groups (animals receiving a single injection of non-modified glycopeptides) comes from that of the purification procedure (that is, difference of chemical properties), because all morphologic 


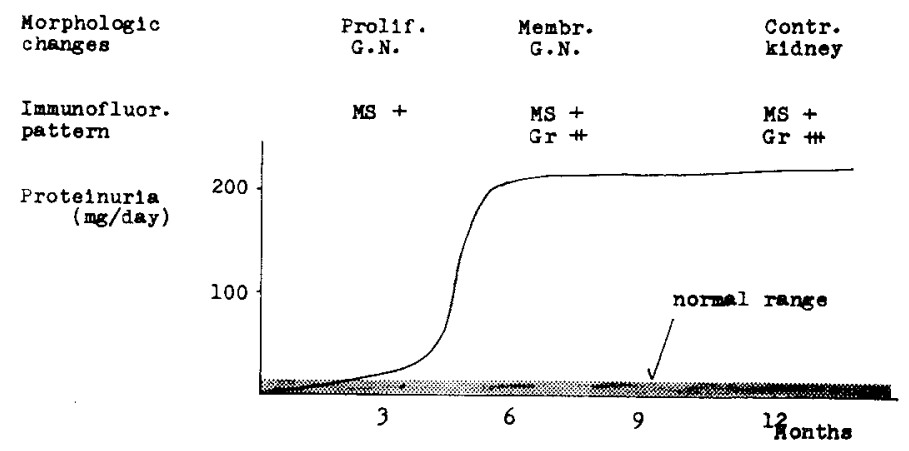

1. Material: Before TCA or DEAE-Cellulose treatment.

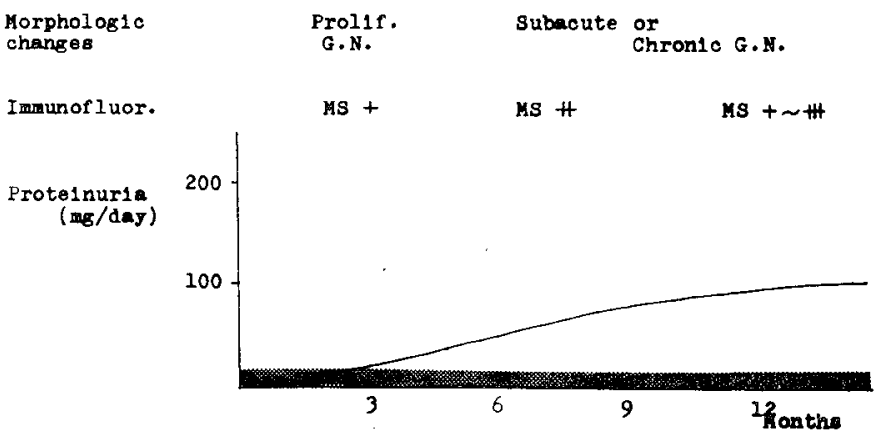

2. Material: After TCA or DEAE-Cellulose treatment.

Fig. 4. Glycopeptide nephritis.

changes of our laboratory model were proved to be induced by a single injection of the known chemical substance.

As shown in upper half of Fig. 4, morphologic changes of membranous glomerulonephritis developed slowly (3 to 4 months) after a single injection of nephritogenic glycopeptide (non-modified), and at the same time, marked proteinuria over $200 \mathrm{mg}$ per day manifested and regular granular deposition of $\gamma$-globulin and $\beta_{1} \mathrm{C}$ accomplished along the GBM, besides mesangial deposits of protein which is the basic immunofluorescent pattern of this laboratory model.

In addition, rabbit antisera against the nephritogenic glycopeptide did not contain kidney-fixing antibody.

Considering with these results, it seems likely that antigen-antibody immune complex mechanism may accelerate or intensify the glomerular damage which was induced by basal mechanism of this laboratory model. 


\section{Conclusion}

It can be concluded that basal immunofluorescent staining patterns of the glomerular capillary walls of this experimental glomerulonephritis is so-called "mesangial pattern", and membranous glomerulonephritis with immunofluorescent granular pattern is only one of subtypes of the spectrum of basal glomerulonephritis which was induced by a single injection of nephritogenic glycopeptide.

In addition, it is of particular interest that basal immunofluorescent pattern of this laboratory model was far different from the linear pattern which is generally produced by anti-GBM antibodies, in spite of the use of the material prepared from GBM.

Mechanism other than "auto-immune mechanism" should be considered for the basic mechanism of this glycopeptide nephritis.

\section{References}

1) Natali, P.G. and Tan, E.M.: Experimental renal disease induced by DNA-anti-DNA immune complex. J. Clin. Invest. 51:345, 1972.

2) Koffler, D., Schur, P.H. and Kunkel, H.G.: Immunological studies concerning the nephritis of systemic lupus erythematosus. J. Exp. Med. 126: 607, 1967.

3) Koffler, D., Agnello, V., Carr, R.I. and Kunkel, H. G.: Variable patterns of immunoglobulin and complement deposition in the kidneys of patients with systemic lupus erythematosus. Am. J. Path. 56:305, 1969.

4) Gallo, G. : Elution studies in kidneys with linear deposition of immunoglobulin in glomeruli. Am. J. Path. 61:377, 1970.

5) Shibata, S., Naruse, T., Nagasawa, T., Takuma, T. and Miyakawa, Y.: Purification by starch block electrophoresis of renal antigen that induces nephrotoxic antigen. J. Immunol. 99 : 454, 1967.

6) Shibata, S., Miyakawa, Y., Naruse, T., Nagasawa, T. and Takuma, T.: A glycoprotein that induces nephrotoxic antibody: its isolation and purification from rat glomerular basement membrane. J. Immunol. 102: 593, 1969.

7) Shibata, S., Naruse, T., Miyakawa, Y. and Nagasawa, T.: Further purification of the glycoprotein that induces nephrotoxic antibody: isolation of the active polysaccharide fraction mainly composed of glucose. J. Immunol. $104: 215,1970$.

8) Shibata, S., Nagasawa, T., Naruse, T. and Miyakawa, Y.: Glomerulonephritis induced in rats by antiserum against a glycoprotein from rat kidney-A new experimental model of glomerulonephritis. Jap. J. Exp. Med. 37: 337, 1967.

9) Shibata, S., Nagasawa, T., Miyakawa, Y. and Naruse, T.: Nephritogenic glycoprotein I. Proliferative glomerulonephritis induced in rats by a single injection of the soluble glycoprotein isolated from homologous glomerular basement membrane. J. Immunol. 106 : 1284, 1971 :

10) Shibata, S., Sakaguchi, H., Nagasawa, T. and Naruse, T.: Nephritogenic glycoprotein II. Experimental production of membranous glomerulonephritis in rats by a single injection of homologous renal glycopeptide. Lab. Invest. $27: 457,1972$.

11) Shibata, S., Sakaguchi, H. and Nagasawa, T.: Nephritogenic glycoprotein V. Experimental chronic glomerulonephritis induced in rats by a single injection of homologous renal glycopeptide. in preparation.

12) Shibata, S.: A new laboratory model for adult human progressive glomerulonephritis. Proceedings of 8 th international congress of allergology, p. 278, 1974 Excerpta Medica. 